\title{
RESEARCH OF PROBABILITY CHARACTERISTICS IN DEFECT DETECTION OF COMPOSITE MATERIALS USING WAVELET TRANSFORM
}

\author{
V.V. Gerasimov, V.S. Khandetsky, S.N. Gnoevoy \\ Nauchnaya str., 9 Radio-physics department Dnipropetrovsk National University, \\ Dnipropetrovsk, Ukraine 49000, E-mail: gerasimov@mail.dsu.dp.ua
}

\begin{abstract}
Composite materials with carbon reinforced fibres now are widely applied in aircraft and space techniques. The influence of roughness to sensor signal is amplified and becomes comparable and in some cases more amplitude of a modulation pulse of a surface defect. Statistical and probability researches spectral and wavelet methods of identification have been done. Methods have been tested on more than two millions copies of signals of surface cracks.
\end{abstract}

Keywords: Defectoscopy of composites, Fourier transformation, Wavelet transform

\section{Introduction}

Composite materials with carbon reinforced fibres now are widely applied in aircraft and space techniques. Carrying carcasses and heat-shielding coverings are produced using these materials. Taking into account conducting properties of such materials which in addition amplify after high-temperature thermal processing, for search and an estimation of geometrical parameters of cracks it is expedient to use a contactless method of eddy currents. The surface of products from fibrous materials is characterized by significant roughness usually. By reason of influence of some uncontrollable factors during manufacturing and heat treatments of a product the wisps of fibres can non-uniformly settle down and the relief of the product's surface gets stochastic character. Necessity of increase in sensitivity of eddy current sensor to shallow and short surface cracks that break one or two layers of carbon fibres results to essential reduction of the radial sizes of sensor. That is why the influence of roughness to sensor signal is amplified and becomes comparable and in some cases more amplitude of a modulation pulse of a surface defect. The process of defect detection is considerable complicates by lift-off or tilt effects of eddy-current sensor.

\section{Signal models and identification methods}

\subsection{Signal models}

Basing to results of the previous researches, modulation signal of surface crack was described by superposition of gauss's curves 


$$
s_{1}(t, k)=e^{-3 t^{2} / 2}-k e^{-3 t^{2}}, k \in[0,1]
$$

signal lift-off or tilt effect of eddy-current sensor — by signal of paraboloidal type

$$
s_{2}(t, q)=2 \cdot\left(1-2^{\lambda} \operatorname{ch} \lambda t\right)
$$

where $\lambda=2.7 q-3$.

The parameter $q$ characterizes speed of moving of the sensor and equals 1 in case of uniform movement; therefore in present article this elementary case is investigated. In this case the deviation of a signal $s_{2}(t, 1)$ from a parabola is not more $1 \%$. The signal $s_{1}(t, 0)$ passes in a gauss's curve and corresponds to long cracks (in comparison with sensor diameter). The signal $s_{1}(t, 1)$ describes a signal of dot defect.

On fig. 1 these signals with $k=1$ and $q=1$ are shown to which is added additive white gauss's noise with a standard deviation $\sigma=0.2$.
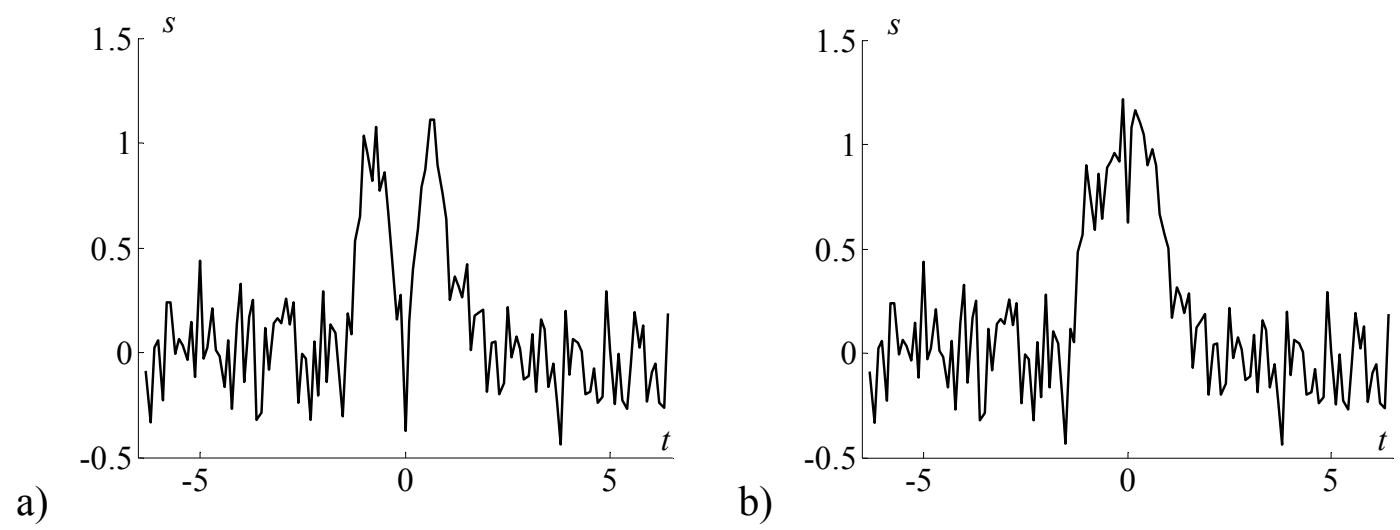

Fig. 1: Signals $s_{1}(t, 1)$ and $s_{2}(t, 1)$ with additive noise $(\sigma=0.2)$.

\subsection{Identification method based on the classical Fourier transformation}

Eddy currents defect detection of composite materials is characterized by small quantity of points of a signal of surface crack, therefore in view of convenience of realize of fast Fourier transformation, samples with the signals normalized on the maximal value $s_{1}$ and $s_{2}$ for researches have been taken in 32 points. For reception of more detailed spectrum signals were supplemented on the right and at the left with zero points up to 128 points. The received spectrum of a pure signal $s_{1}(t, 1)$ (fig. 2a) correspond to the spectra received as a result of experimental researches $[1,2]$ that testify to adequacy of modulation signal used model of crack. The analysis of spectra of pure signals (fig. $2 a, b$ ) has shown expediency of use identification parameter that describe distribution of signal capacity in the most informative zone of lowfrequency area of a spectrum $0 \div 0.1$ (normalized on sampling rate), as in a range approximately $0.05 \div 0.1$ the amplitude of harmonics of a signal $s_{1}(t, 1)$ is over amplitude of harmonics of a signal $s_{2}$. The amplitude of harmonics of a signal $s_{1}(t, 0)$ on the contrary is less than amplitudes of harmonics of a signal $s_{2}$.

For identification a ratio factor $K_{h}$ of the amplitudes sum of harmonics 6 to 14 (range of frequencies $0.047 \div 0.11$ ) to the sum of amplitudes of harmonics 1 to 14 was used.

\subsection{Identification method based on the wavelets transform}

The direct wavelets transform (continuous transformation) means decomposition of a signal with use of basis as set of wave packages - wavelets which are characterized by the following features $[3,4]$ :

- look like short, located in time (or in space), wave packages with zero value of integral;

- have an opportunity of shift on time; 
- $\quad$ are scalable (can be compress / stretch);

- have limited (or local) frequency spectrum.

This basis can be orthogonal, but there are wavelets which do not have properties of orthogonality, but are practically useful. Wavelet transform of function $f(t)$ is a function

$$
W(s, x)=\frac{1}{\sqrt{s}} \int_{-\infty}^{\infty} f(t) \psi\left(\frac{t-x}{s}\right) d t
$$

where $\psi(t)$ is the base wavelet.

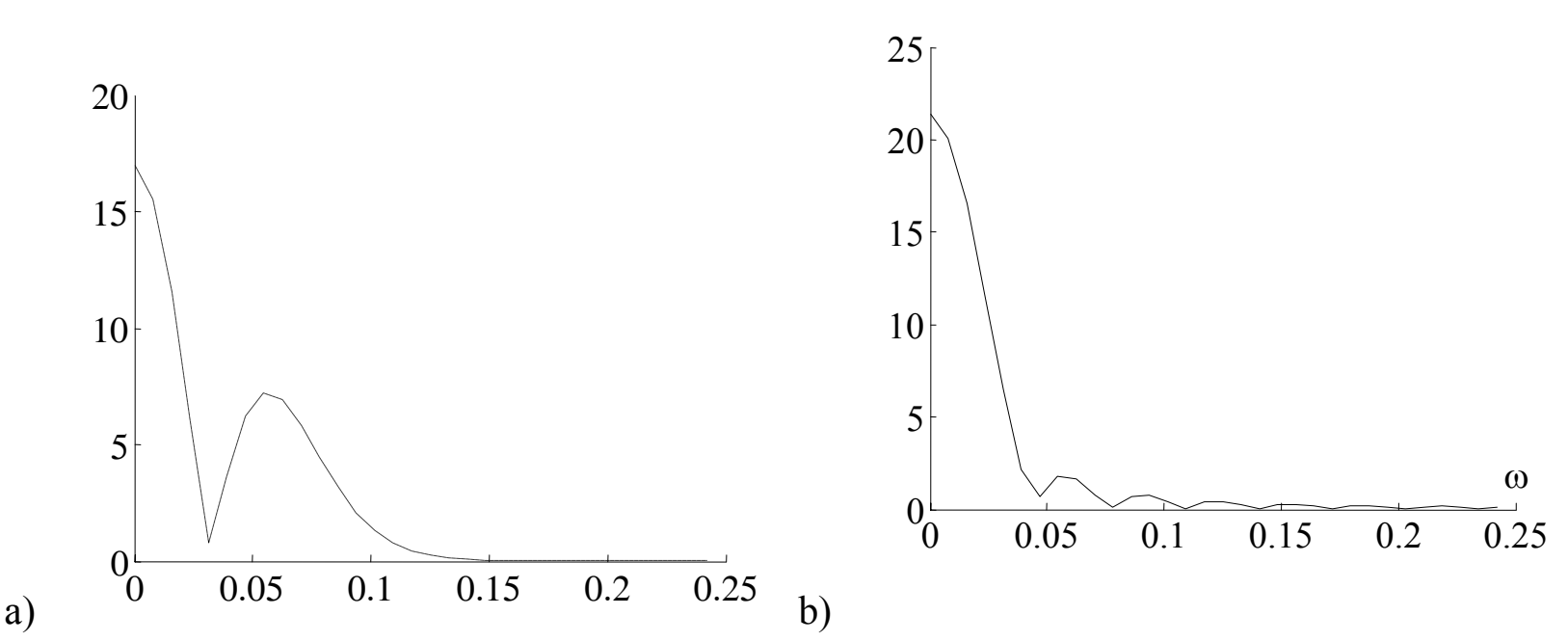

Fig. 2: Spectrums of signals: a) $s_{1}(t, 1)$; b) $s_{2}(t, 1)$.

Wavelet transform is function of two variables: scale $s$ and shift $x$. It is decomposition of a signal on all possible shifts and stretching/compression base wavelet. As amplitude understand value $W, x$ represents time shift wavelet, $s-$ scale of wavelet. By analogy with Fourier transformation the increase scale $s$ in wavelet transform corresponds to reduction of frequency $\omega$ in Fourier transformation. Display of signal features depends on a kind basic wavelet.

Considering features of analyzed signals (1) and (2) we have chosen gauss's wavelet second order $g_{2}(x)=\left(1-x^{2}\right) e^{-x^{2} / 2}$.

On fig. 3 wavelet spectra of noised signals of surface crack and lift-off/tilt of eddy current sensor (fig. 1) are represented. The $\mathrm{Y}$-axis represent the scale of transformation $s$, the $\mathrm{X}$-axis represent displacement of a signal $x$. Dark zones on diagrams answer to zero values of factors $W$; the brightness is proportional to the module of factors. Regard must be paid to that $x=64$ corresponds to a time point $t=0$.

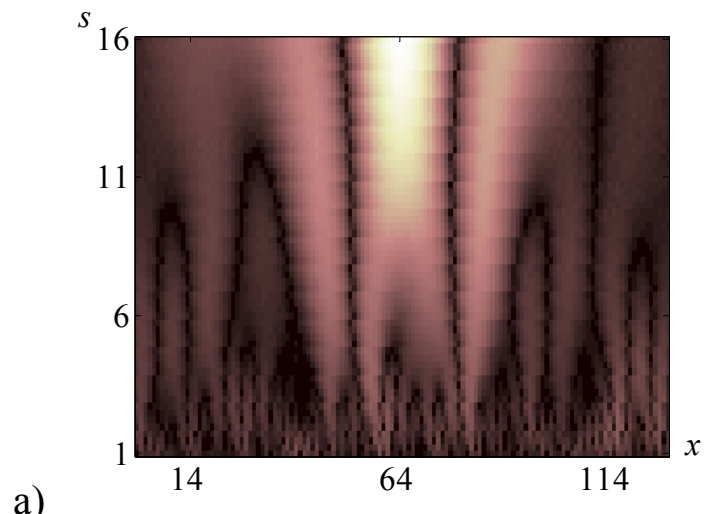

b)

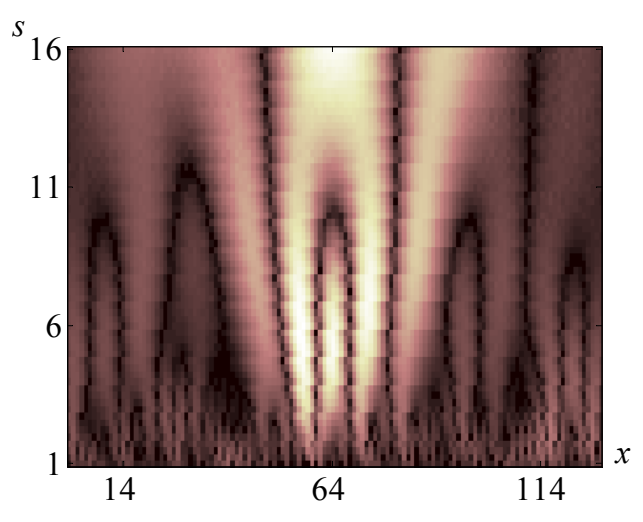


Fig. 3: Wavelet spectra: a) $s_{1}(t, 1)$; b) $s_{2}(t, 1)$.

The analysis of the represented wavelet spectrograms has shown expediency of use for signal identification of factor value of wavelet-decomposition in the central area at small scales as in this area the greatest changes $W(s, x)$ are observed.

For an estimation of distribution $W(s, x)$ on condition of action of noise we suggest to use information parameter as:

$$
K_{w}=\int_{s_{r}-h}^{s_{r}+h} \int_{x_{0}-w}^{x_{0}+w} W(s, x) d x d s
$$

where $h, w$ - the sizes of area on scale $s$ and shift $x$.

The analysis of wavelet-spectrograms of signals (1) and (2) has shown that the maximum of the information for signal identification can be received using area of integration $4 \leq s \leq 12$ and $60 \leq x \leq 68$.

For signal identification the factor $K_{w}$ received by summation of factors $W(s, x)$ in the area determined above has been used.

\section{Comparative research}

\subsection{Statistic analysis}

The capacity of identification of signals of surface cracks with $k=0 \ldots 1.0$ with step 0.1 has been investigated. Ability of identification of signals of surface crack (1) and signal of lift-off/tilt sensor (2) was investigated. A standard deviation of noise $\sigma$ was 0.1 to 2 with step 0.02 (the modulation impulse is normalized on the maximal value). For each signal, at each value of a standard deviation of noise 1000 tests have been done.

By the received results histograms of occurrence of corresponding information parameters $K_{h}$ and $K_{w}$ are created and corresponding functions of probability density are received.

For carrying out of the qualitative analysis of signal identification the average values of information parameters have been used (fig. 4).

On the above-stated graphs the central thicker lines is the average value of identification parameter, more thin lines are ranges of standard deviation of parameter.

The analysis of the received dependences of average values of identification parameters from a standard deviation of noise has shown that for a spectral method of identification average value of parameter $K_{h}$ strongly depends from $\sigma$ - with increase $\sigma$ average value of parameter $K_{h}$ for the signal of lift-off/tilt increases and comes nearer to value of parameter for the signal of surface crack. For wavelet-method of identification average value of parameter $K_{w}$ practically does not depend from $\sigma$, the small increase in non-uniformity of average value about increase $\sigma$ is observed only. Increase noise increases standard deviation of parameters $\sigma_{K}$ - sets of parameters begin to be crossed for the spectral method at $\sigma=0.55$ and for the wavelet-method at $\sigma=0.9$.

Further dependences of average value of identification parameters from length of surface crack (parameter $k$ ) have been analysed. For the spectral method the line of average value $K_{h}$ for crack signal comes nearer to the line $K_{h}$ of the signal of lift-off/tilt at increase the parameter $k$ from 0 to 0,5 . Further with increase $k$ from 0,5 to 1,0 line of average value for crack signal keeps away from the line of signal of lift-off/tilt of sensor.

For the wavelet-method dependence of identification parameter on length of the crack is monotonous. For this method the line $K_{w}$ of average value of crack signal comes nearer to the line $K_{w}$ of average value for lift-off/tilt of sensor at increase in parameter $k$ from 0 to 0.65 , then crosses it and at $k=1$ reaches the maximum. Regard must be paid to the presence of a zone of tolerance of wavelet-method - this method is not capable to differentiate signals (1) with $k \approx$ 
0.65 from signals of a kind (2), i.e. parameter $K_{w}$ for surface cracks of the certain length will be same, as well as for the signal of lift-off/tilt. Also, as well as the spectral method, this method has zone of uncertain recognition of signals in range $0.5<k<0.8$.

a)

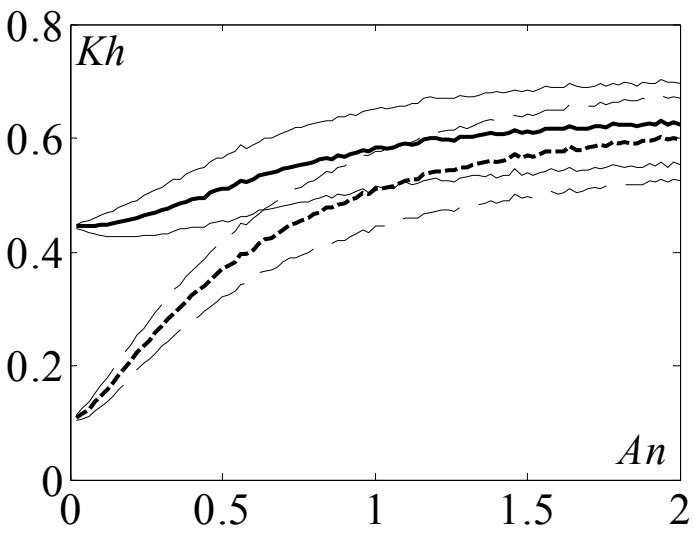

$0 . 5 \longdiv { K h }$

c)
0.4

0.3

0.2

0.1

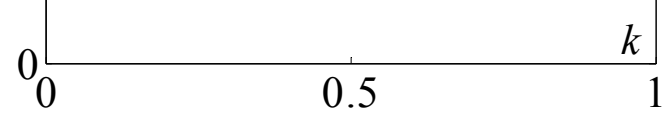

b)
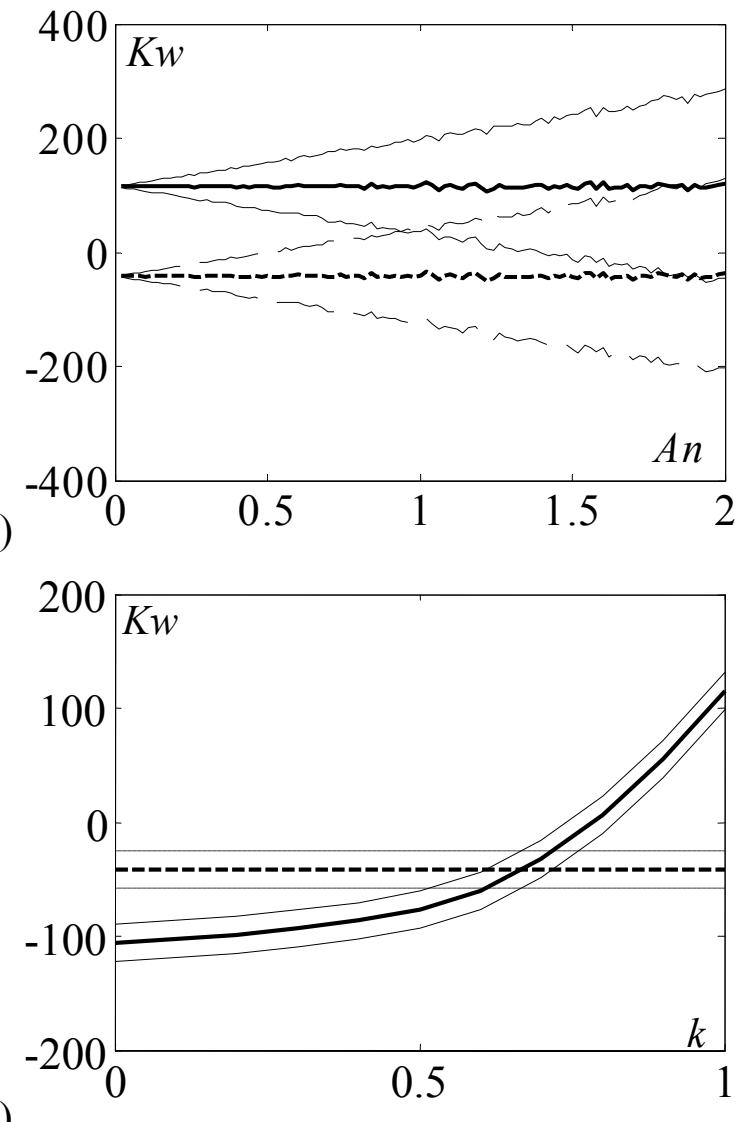

d)

Fig. 4: Average values of identification parameters: a), c) spectral method; b), d) wavelet method; a), b) dependence from $\sigma ; \mathrm{c})$, d) dependence from $k$ : solid line — crack signal; dotted line - signal of lift-off or tilt effect.

For both methods for identification process it is necessary to choose a threshold concerning which the decision on classification of a signal as the crack signal or the signal of lift-off/tilt will be accepted. As such threshold it is possible to choose average value of two average identification parameters for crack signal $s_{1}(t, 1)$ and the signal of lift-off/tilt $s_{2}(t, 1)$. Thus for a spectral method of identification if threshold value will not allow noise level, at $\sigma \approx 0.3$ a part of signals of lift-off/tilt of sensor will be identified as signals from the surface crack. If threshold value will be corrected depending on noise level, the area of correct identification will be expanded to $\sigma \approx 0.6$. Thus only cracks with $\mathrm{k}>0.8$ (short cracks) will be identified. If for wavelet-method to use in the same way chosen threshold it can be not corrected depending on noise level. With increase in noise level $(\sigma>1)$ only will monotonously increase probability of false identification.

\subsection{Probability analysis}

Further graphs of identification probability at the set probability of a false alarm for crack signals with $k=0,0.5,0.7,1.0$ have been created. Values $k=0.5$ and $k=0.7$ have been chosen specially as at such parameters average values of functions of probability density for crack signals and signals lift-off/tilt of sensor are maximum close (fig. $4 c, d$ ). 
The received figures (performance characteristics) differ only in degree of curvature (fig. 5a shows one variant, $k=0.5$ ). Therefore for the qualitative analysis of these characteristics it is possible to use one characteristic point. It can be chosen as follows. First, it is possible to fix probability of recognition of the signal or probability of the false alarm. Second, it is possible to choose an inflection of the line that is the point which the tangent of an angle of slope of a tangential line is equal 1 - it is a characteristic point which the probability of a false alarm and probability of the miss of a crack are equal. At the equal price of a false alarm and the miss of a crack at equal probabilities of their occurrence in this point there will be optimum economic effect. Using the second way the graph of dependence of identification probability of a crack from parameter $k$ has been created at equality of probabilities of a false alarm and the miss cracks (fig. 5b).

This dependence (fig. 5b) shows how the probability of signals recognition will change at various parameter $k$. Both methods have a zone of bad probability of recognition of signals at $k=0.4-0.8$. For spectral method this area is wider, than for wavelet method. In other range of values $k$ the wavelet method have better results over the spectral method.

a)

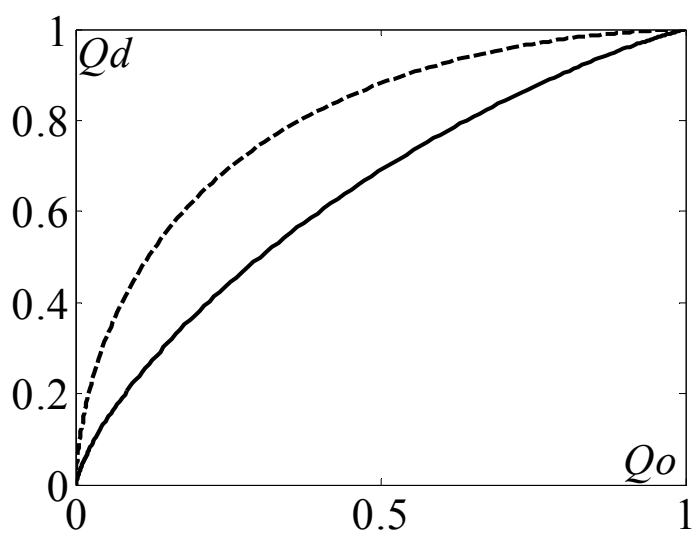

b)

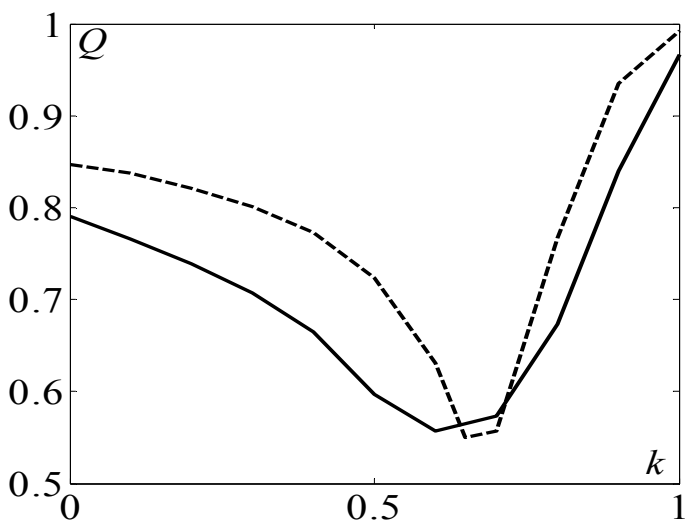

Fig. 5: Performance characteristic (a) and probability of signal identification (b): solid line - spectral method; dotted line - wavelet method.
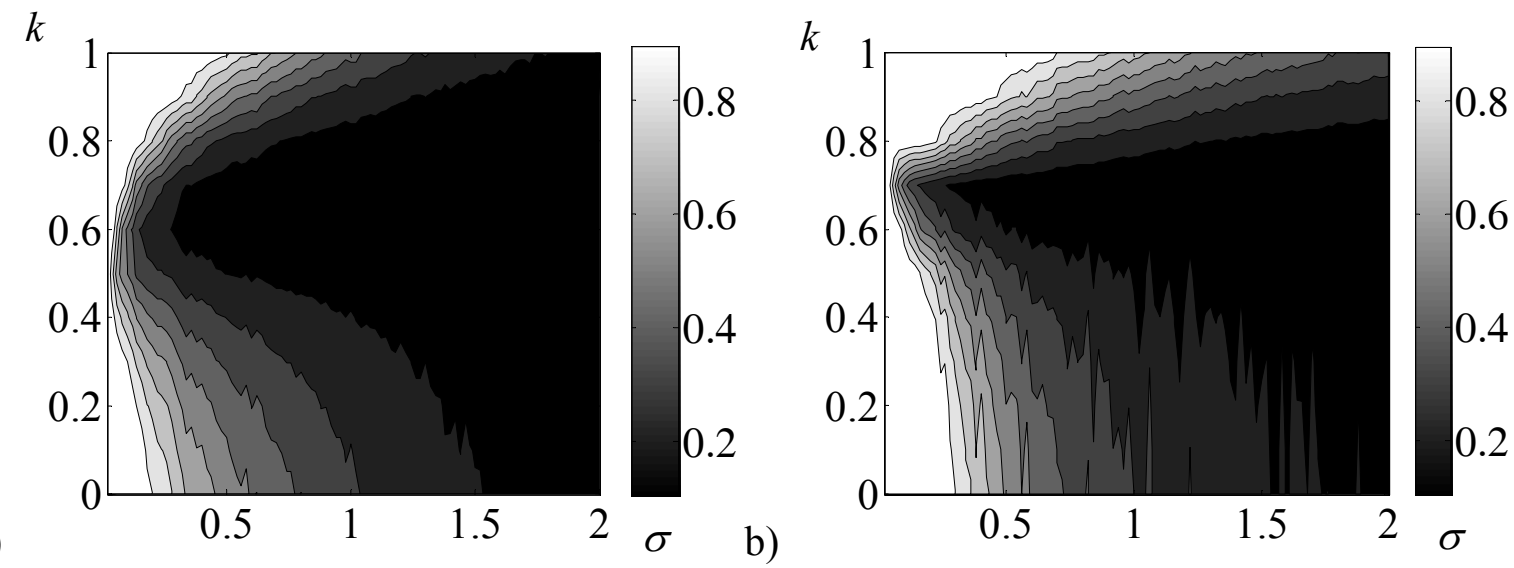

Fig. 6: Planimetric maps: a) spectral method; b) wavelet method.

The graph represented on fig. $5 \mathrm{~b}$ gives us value of recognition of crack signals for $\sigma=0.4$, but dependence of probability of crack signal recognition from a standard deviation of noise $\sigma$ is so interesting. For creation such dependence, as initial we choose dependences of probability of 
recognition of crack signal on probability of a false alarm at various $\sigma$. Further we determine probability of recognition of crack signal at the fixed probability of false alarm of equal $10 \%$ (characteristic points it is chosen by the first way). We received dependence of probability of recognition of crack signal on parameter $k$ and standard deviation $\sigma$. For display of such dependence we used planimetric maps (fig. 6).

These planimetric maps give more full representation about an opportunity of identification of signals of surface cracks and signals of lift-off/tilt of the sensor on the background gauss's noise. Both methods uncertainly distinguish surface cracks with $0.55<k<0.75$. More steadfast analysis of these maps shows, that the wavelet-method has the best identifying ability, than the spectral method, especial in the area of short cracks. Signals from short surface cracks with probability 0.8 spectral method are distinguished at noise level about $60 \%$, wavelet-method at noise level up to $100 \%$ from amplitude of a signal.

\section{Conclusions}

Statistical and probability researches spectral and wavelet methods of identification have been done. Methods have been tested on more than two millions copies of signals of surface cracks and lift-off/tilt of sensor. Signals with a standard deviation of noise $\sigma=0.1 . .2$ were used.

By results of researches the following features of definition of threshold value for parameter of identification have been revealed:

- for improvement of quality of identification the threshold for spectral method should consider noise level;

- for wavelet method at definition of identification the threshold noise level is not important. Both methods have shown the best ability to identification short cracks. Generally the wavelet method has the best ability to signal identification, but it is more complex and therefore in case of small noise the spectral method can be the best decision due to higher speed.

\section{References}

[1] Khandetsky V.S., Grechka A.T. Spectral diagnostic of continuity of carbon composite. Mechanics of composite materials. 1989. № 5. P. 922-924.

[2] Khandetsky V.S. Sopilnik A. V., Grechka A. T. Detection the surface cracks in carbon composite. // Defectoscopy. 1994. № 2. P. 47-57.

[3] Mandrykova O. V., Tristanov A. B. Research the local features of time series by Wavelet Toolbox. // Papers Russian science conference "Design science and engineering applications in MATLAB". M.: IPU RAS, 2004. P. 242-260.

[4] Tristanov A. B. Detection the changes in signal by wavelet method. // Papers Russian science conference "Design science and engineering applications in MATLAB". M.: IPU RAS , 2004. P. 1798-1821. 